\title{
The ensemble particle filter (EnPF) in rainfall-runoff models
}

\author{
G. van Delft · G. Y. El Serafy · A. W. Heemink
}

Published online: 13 January 2009

(C) Springer-Verlag 2008

\begin{abstract}
Rainfall-runoff models play a very important role in flood forecasting. However, these models contain large uncertainties caused by errors in both the model itself and the input data. Data assimilation techniques are being used to reduce these uncertainties. The ensemble Kalman filter (EnKF) and the particle filter (PF) both have their own strengths. Research was carried out to a possible combination between both types of filters that will lead to a new type of filters that joins the strengths of both. The so called ensemble particle filter (EnPF) new combination is tested on flood forecasting problems in both the hindcast mode as well as the forecast mode. Several proposed combinations showed considerable improvement when a hindcast comparison on synthetic data was considered. Within the forecast comparison with field data, the suggested EnPF showed remarkable improvements compared to the PF and slight improvements compared to the EnKF.
\end{abstract}

Keywords Rainfall-runoff models - Ensemble

Kalman filter · Particle filter - Ensemble particle filter

\section{Introduction}

Rainfall-runoff (RR) models are widely used in flood forecasting. Governments throughout the world are interested in flood forecasts. When accurate forecasts are available one might be able to respond to upcoming high

G. van Delft · A. W. Heemink

Delft University of Technology, Delft, The Netherlands

G. Y. El Serafy $(\square)$

Deltares, Rotterdamseweg 185, 2629 HD Delft, The Netherlands

e-mail: ghada.elserafy@deltares.nl water levels. Governments might issue orders to, temporarily, raise the dikes to prevent a country from flooding or in extreme floods to evacuate.

A lot of uncertainties are involved in flood forecasting. First the model used to forecast the flood is never perfect. The model is a simplification of the real world process and thus contains errors. Weather forecasts are needed in order to make any prediction on the flood. For example, if there is no information and/or forecast of the amount of precipitation for the upcoming days, it will be impossible to predict the water level accurately.

From time to time measurements of the water level are available. These measurements can be combined with predictions obtained from the model. Through data assimilation the system state (i.e. model variables), and thus the model predictions, can be improved.

Various data assimilation methods exist. The Kalman filter probably being the most well known. The original Kalman Filter, as introduced by Kalman (1960), will not be considered here, because of its limitation to linear models.

The ensemble Kalman filter (EnKF) and particle filters (PF) are both suitable for non-linear models. The ensemble Kalman filter was introduced by Evensen (1994). The particle filter was introduced by Isard and Blake (1998).

The PF can represent any kind of posterior probability density function, where the EnKF will assume all errors to be Gaussian distributed. This is considered as an advantage when working with RR models. One of the disadvantages of the PF would be that during the update step, no real update is being carried out. Resampling can cause filter failure (degeneracy) when the model output and/or state "domain" are far from the measurements domain (ref). The EnKF scheme on other hand will minimize the variance of the ensemble members. And thus all ensemble members will be updated by the EnKF. 
Numerous publications concerning practical application of both the EnKF and PF are available. Among others, French et al. (1992) address the need and the benefit of using the Monte Carlo simulation for addressing the uncertainty in rainfall fields and its forecasting process. Shu et al. (2005) use the ensemble Kalman filter rainfall runoff hydrological models to improve the estimate of the soil moisture. Several applications on the flood forecasting field have previously been studied, Weerts and El Serafy (2006) compare the ensemble Kalman filter and the particle filter, and Koster et al. (2004) study the effect of input correction on the conceptual rainfall runoff model used in this paper (i.e. Hymod model). This paper will study how to combine both filtering algorithms into a new filter: the ensemble particle filter (EnPF). This new filter should combine the best of both filters.

In literature, some first thoughts about possible combination have already been discussed. Mandel and Beezley (2006) introduced a new algorithm that exists of three steps: the prediction, the proposal and the update. This means first the EnKF will be applied, followed by a resampling stage from the $\mathrm{PF}$. This suggestion is studied among some other proposed ideas for a combination. These ideas of combinations will be tested on the conceptual rainfall runoff model (Hymod model).

\section{HYMOD model}

A description of the rainfall runoff model is first given in Sect. 2.1. The model is applied to the setting of the Leaf River watershed in Mississippi (USA). A dataset containing daily precipitation, evapotranspiration, and discharge observations was obtained from the National Weather Service Hydrology Laboratory (HRL). This field dataset will be used as reference for comparisons throughout the rest of this paper.

\subsection{Model description}

The HYMOD model is a non-linear rainfall-runoff conceptual model of which the general concepts are based on an article by R. J. Moore (1985). It relates the mean areal precipitation and the potential evapotranspiration, for a certain catchment, to the discharge. A detailed description of the HYMOD model was given by Bos and De Vreng (2006). Here a short overview will be presented.

The model consists of two components, a slow flow component and a quick flow component. The slow flow component consists of one linear reservoir, and represents the water that flows through the ground and eventually ends up in the river. The quick flow component consists of three identical quick flow reservoirs. This part represents the water that flows directly into the river.

The model uses two input variables: mean areal precipitation, $P$ ( $\mathrm{mm} /$ day), and potential evapotranspiration, $E$ ( $\mathrm{mm} /$ day). Both are daily averaged observations from meteorological stations over a certain catchment's area as obtained from the National Weather Service Hydrology Laboratory (HRL). The first stage of Fig. 1 represents the catchments storage capacity, with maximum storage capacity $C_{\max }$. The excess precipitation, $U_{1}$, will directly flow, through the quick flow tanks, into the river. Depending on the catchment's soil moisture capacity $b_{\exp }$ a certain amount of water, $U_{2}$, will flow to either the slow or the quick flow component. The factor $\alpha$ represents the percentage of the amount of water of $U_{2}$ that will flow to the quick flow component. From the quick and slow flow tanks, $x_{\text {quick } 1}, x_{\text {quick2 }}, x_{\text {quick } 3}$, and $x_{\text {slow }}$, respectively, a fraction of the water will flow to either the next flow tank, or into the river $(Q) . R_{\mathrm{q}}$ and $R_{\mathrm{s}}$ are coefficients representing those fractions for the quick and slow flow components, respectively.
Fig. 1 A schematic overview of the HYMOD rainfall-runoff model

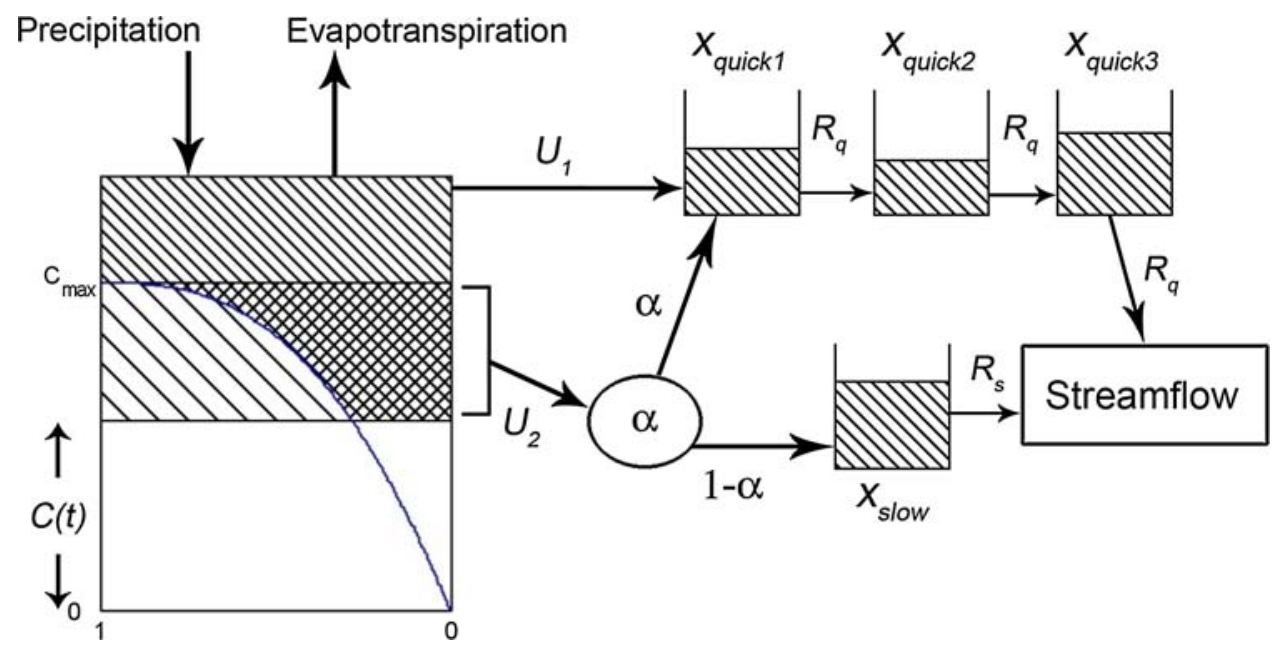




\subsection{Sensitivity analysis}

To get a better understanding of the hydrological HYMOD model, the effect of the changes in the state (i.e. model variables), input variables (i.e. driving forces), and the parameters on the model output is carried out through a sensitivity analysis. Perturbations were one by one added to each of the model states, inputs and certain parameters. This sensitivity analysis is very important when defining the state used in filtering (i.e. here addressed as filter state). Since in the rainfall runoff conceptual model, the driving forces (i.e. precipitation and evapotranspiration), are the governing variables in the physical process and thus on the model output (i.e. discharge), the results of the sensitivity analysis are presented in this paper for the driving forces only.

For each day perturbations are added to the each driving force separately and the results on the model output is being studied. The standard deviation of the perturbations was chosen as a combination of a fixed value and a percentage of the actual input value for precipitation or evapotranspiration, respectively as in Koster et al. (2004). Figure 2 shows the effect of perturbations in the precipitation and evapotranspiration separately on the discharge. During low discharge the perturbation induced on the evapotranspiration has no significant effect on the discharge, while during high discharge the perturbation induced on the precipitation has much more pronounced effect on the discharge than those of the evapotranspiration on the discharge. Since the model is more sensitive to precipitation compared to evapotranspiration, for the case that was considered, no evapotranspiration was considered as part of the filter state. By not considering the evapotranspiration in the filter state, the filter in the update step will attach all the difference between the model and the measurements to changes or corrections in the rainfall only and not in the evapotranspiration.

This sensitivity analysis has shown that for the specific situation that has been studied (i.e. the Leaf River area) the precipitation is the most significant governing driving force for the discharge. An $A R(1)$ process that will be used in order to model the errors on precipitation. This has been thoroughly discussed by Koster et al. (2004). This results in the following state vector,

$X^{k}=\left[\begin{array}{llllll}\delta U^{k} & x_{\text {loss }}^{k} & x_{\text {slow }}^{k} & x_{\text {quick }_{1}}^{k} & x_{\text {quick }_{2}}^{k} & x_{\text {quick }_{3}}^{k}\end{array}\right]^{\prime}$

where $\delta U^{k}$ represents the variation on the precipitation to be estimated. This state-space representation will be used by the EnKF, the PF and the proposed combination filters throughout the paper.

\section{Theory}

Detailed descriptions of the Ensemble Kalman Filter and the Particle filter are respectively given by Evensen (2003)
Fig. 2 The effect of daily perturbations to both precipitation (top panel) and evapotranspiration (bottom panel) on the discharge. The black line corresponds with the discharge for unperturbed inputs
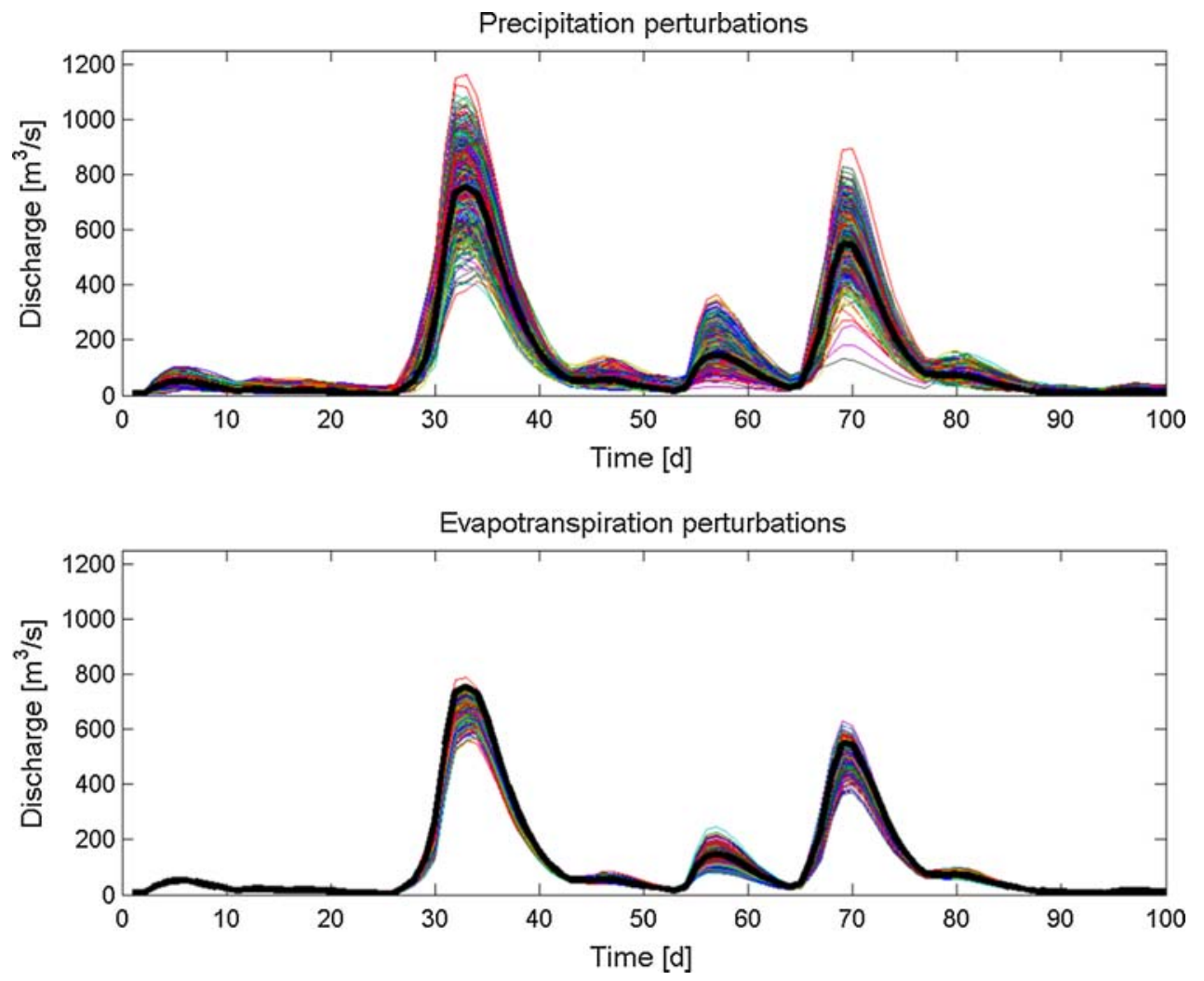
and Isard and Blake (1998). This section will provide short descriptions of the two existing filter algorithms. Furthermore, the proposed combinations ensemble particle filter $(\mathrm{EnPF})$ are fully described in Sect. 3.3.

\subsection{Ensemble Kalman filter}

All the filters that will be discussed in this section will start with the time-update of the model. In case of the EnKF this means that all ensemble members $(i=1, \ldots, N)$ will be forwarded into time

$\bar{X}_{i}^{k+1}=f\left(\hat{X}_{i}^{k}\right)+W_{i}^{k+1}$

$Y_{i}^{k+1}=g\left(\bar{X}_{i}^{k+1}\right)+V_{i}^{k+1}$

where $W_{i}^{k+1}$ represents a white Gaussian model noise process with zero mean and covariance matrix $Q^{k+1}$, while $V_{i}^{k+1}$ represents the white measurement noise process with zero mean and covariance matrix $R^{k+1}$.

The ensemble mean of the state vector and the measured state are calculated

$\bar{X}^{k+1}=\frac{1}{N} \sum_{i=1}^{i=N} \bar{X}_{i}^{k+1}$

$\overline{g\left(X^{k+1}\right)}=\frac{1}{N} \sum_{i=1}^{N} g\left(\bar{X}_{i}^{k+1}\right)$

The Eqs. 4 and 5 have been discussed by Houtekamer and Mitchell (2001) and allow for non-linear measurement functions in the calculation of the Kalman gain matrix from the ensemble. The following terms which represent the state covariance projected in the measured location and the measured state covariance matrix, respectively.

$\left(P H^{\prime}\right)^{k+1}=\frac{1}{N-1} \sum_{i=1}^{N}\left(X_{i}^{k+1}-\bar{X}^{k+1}\right)\left(g\left(X_{i}^{k+1}\right)-\overline{g\left(X^{k+1}\right)}\right)^{\prime}$

$$
\begin{aligned}
\left(H P H^{\prime}\right)^{k+1}= & \frac{1}{N-1} \sum_{i=1}^{N}\left(g\left(X_{i}^{k+1}\right)-\overline{g\left(X^{k+1}\right)}\right) \\
& \times\left(g\left(X_{i}^{k+1}\right)-\overline{g\left(X^{k+1}\right)}\right)^{\prime}
\end{aligned}
$$

Finally, the Kalman gain matrix is determined as follows, and to update all ensemble members

$K^{k+1}=\left(P H^{\prime}\right)^{k+1}\left(\left(H P H^{\prime}\right)^{k+1}+R^{k+1}\right)^{-1}$

while the ensemble members are updated with the ensemble of measurements as follows

$\hat{X}_{i}^{k+1}=\bar{X}_{i}^{k+1}+K^{k+1}\left(Y_{i}^{k+1}-g\left(\bar{X}_{i}^{k+1}\right)\right)$

Note that the time-update, Eqs. 4 and 5, will consist of both the HYMOD model update as well as the update for the $A R(1)$ process. This will give some difficulties for the EnKF to provide an estimate for the precipitation.

The state vector $X_{i}^{k}$, in Eq. 2, contained the precipitation variation $\delta U_{i}^{k}$ (compare with Eq. 1). When the time-update has been carried out, the state vector $\bar{X}_{i}^{k+1}$ contains the precipitation variation $\delta U_{i}^{k+1}$ that will be used by the model in the next time step. This is the value that will be updated during the measurement update of the EnKF. Hence, when working with the EnKF, $\delta U_{n+1}$ is not updated based on the current observations. In order to update the variations on precipitation with the ensemble Kalman filter the time-update should be split into two parts, where the first part would consist of the HYMOD model update and the second part the $A R(1)$ process.

\subsection{Particle filter}

The first stage, the time-update, of the PF is equal to the EnKF (Eqs. 2, 3). The analysis stage of the PF is different though. Weights will be determined corresponding to each of the particles

$\tilde{w}_{i}^{k+1}=p\left(Y_{\mathrm{obs}} \mid g\left(X_{i}^{k}\right)\right)$

where the weights are normalized through the following equation

$w_{i}^{k+1}=\frac{\tilde{w}_{i}^{k+1}}{\sum_{i=1}^{N} \tilde{w}_{i}^{k+1}}$

For this paper, an exponential function was used as weighting function

$p\left(Y_{\text {obs }} \mid g\left(X_{i}^{k+1}\right)\right)=\exp \left(-c_{\exp } \mid g\left(X_{i}^{k+1}\right)-Y_{\text {obs }}\right)$

The calculated weights will now be used in the resampling stage. A discrete probability density functions is constructed, assigning weight $w_{i}$ to particle $i$. From this PDF, $N$ particles will be resampled for the next time step. Different resampling strategies exist. Douc et al. (2005) discusses a number of options for resampling. In this paper, the Stratified Resampling strategy will be used.

In comparison to the EnKF, the PF does not have any problems giving an estimate for the precipitation. The PF will simply calculate the weights corresponding to each of the particles. These weights can than be used to determine the precipitation estimate. No adjustments to the time-update are necessary.

\subsection{Ensemble particle filter combination}

The objective of this paper make is to find an optimal combination between the ensemble Kalman filter and the particle filter. This section will discuss some proposed ideas for a combination. The introduction already referred 
to one possible combination. Other suggestions for the combination include pre-filtering, weighted ensemble Kalman filter and side-by-side EnKF and PF, and a method that uses multiple sample stages.

Pre-filtering considers two separate sets of ensemble members. The first set will be handled as one would do with the EnKF. New estimates for the input values will than be calculated. These (improved) input values will now be used as input values for the driving forces for the second set of ensemble members. This idea on its own does not necessarily mean a combination between the EnKF and the PF. However, it can very well be used in improving the estimate of the PF and avoid filter particle degeneracy and/ or loss of diversity.

The ensemble Kalman filter will normally use a simple mean to calculate the output values. For the particle filter the weights can be used to calculate a weighted average. The weighted ensemble Kalman filter will calculate weights for each of the ensemble members, as is done with the PF, and use these weights to obtain a weighted average as output value.

Another idea was to run the EnKF and the PF side-byside. A certain number of ensemble members will be handled by the EnKF, and the remaining particles will be treated by the PF. One can for example think of an ensemble to which the most likely particles will be added. Numerous variations to this idea are possible. One can choose whether or not there will be interaction between the ensemble members and particles. The particles, for example, can be sampled only from the current particles or from the current particles and ensemble members.

Another proposed combination used multiple sample stages. First a number of particles would be ran through the model, than the corresponding weights would be calculated. These weights would be used to resample the remaining particles. Compared to the original PF this meant more information was used when calculating the weights. Therefore this is expected to improve the quality of the particles. Again this idea does not implicate a combination; this can also be used just for the PF.

This paper will focus on two combinations. The first one uses the ideas of pre-filtering and weighted EnKF. Two ensembles will be used. From the first ensemble a weighted average will be used in order to determine an estimate for the driving forces. These estimated driving forces will be used as input values for the second ensemble. The second ensemble will again be handled with the EnKF. This algorithm will be referred to as $\mathrm{EnPF}_{\mathrm{A}}$.

The second proposed combination that will be discussed in this paper will be using the EnKF and the PF side-byside. Part of the ensemble members will be treated by the EnKF. Weights will be calculated after these ensemble members have been propagated into time. According to these weights a certain amount of particles will be resampled. Thus this combination also uses the idea with multiple sample stages. In the remainder of this paper, this combination will be referred to as $\mathrm{EnPF}_{\mathrm{B}}$.

\subsubsection{EnPF $\mathrm{En}_{\mathrm{A}}$ algorithm}

Assume there are two ensembles consisting of $N_{1}$ and $N_{2}$ ensemble members; total amount of ensemble members equals $N$. For the first ensemble the algorithms is similar to the EnKF; first the time-update for part of the ensemble members, Eqs. 2 and 3. With $i=1, \ldots, N_{1}$. The remaining ensemble members will not yet be dealt with. These ensemble members will be handled as if the EnKF is used. Thus the Eqs. 4-9 are applied to these ensemble members. Next is the combination part of the algorithm. First the weights corresponding to the ensemble members will be calculated compare with Eqs. 10-12 from the PF. These equations will still only be applied to the first $N_{1}$ ensemble members.

The calculated weights can now be used to compute an estimate for the precipitation input. Section 3.1 discussed the advantage of using weights to determine an estimate for precipitation.

From this process one obtains an estimate $\hat{U}^{k+1}$. This estimate will be used in the time-update for the second ensemble

$X_{i}^{k+1}=f\left(\hat{X}_{i}^{k}, \hat{U}_{i}^{k}\right)+W_{i}^{k}$

The remaining part of the algorithms is similar to the Eqs. 2-9, though now applied to the ensemble members $N_{1}+1, \ldots, N$ instead of $1, \ldots, N_{1}$.

\subsection{2 $\mathrm{EnPF}_{B}$ algorithm}

Consider $N_{1}$ ensemble members being handled by the EnKF, and $N_{2}$ resampled particles. For this combination the first part, Eqs. 2-10, is similar to the algorithm for EnPF $F_{\mathrm{A}}$. The second part is different. The weights, as computed in equation, will not (directly) be used to calculate an estimate. Instead they will be used to resample. Weight $w_{\mathrm{i}}$ corresponds with the probability mass for ensemble member $X_{\mathrm{i}}$ (i.e. the ensemble members from the previous time step). Another time-update will be carried out for these resampled particles (Eqs. 2, 3).

\section{Application}

The different data assimilation methods, as have been presented in Sect. 3, will be compared using a synthetic dataset. Field values for precipitation and evapotranspiration will be used as original input values. Perturbations will 
be added to the precipitation input values to obtain the synthetic true precipitation values. The perturbations are normally distributed and having a standard deviation of $15 \mathrm{~mm} /$ day. The perturbed rainfall will be used as input rainfall to the model to determine the true discharge values. On top of the true discharge values measurement errors, also assumed following normal distribution and with standard deviation of $10 \mathrm{~m}^{3} /$ day, will be added to obtain the discharge observations to be used as synthetic data set. Different scales would not affect the results, since in both filters the system noise and measurement noise is also defined using those values (i.e. true values in the generation of synthetic data is used).

First a hindcast comparison will be carried out. The estimated discharge, and the estimated state, will be compared with the known true values for the discharge and state, respectively. In flood forecasting one is obviously more interested in the performance in forecasting mode. The comparison carried out in Sect. 4.2 will focus on forecasting capabilities of the filter.

Both in the hindcast and in the forecast mode comparison, a time span of 3,000 days will be considered. This period of 3,000 days (approx. 8 years) to have a period that is reasonably long and representative for the catchment hydrological behavior with low, high, and moderate runoff. However, both will start with 100 days initialization period. The time step for the model is 1 day; also it is assumed that the observations are available each day.

Experiments indicated the necessity of multiple runs in order to avoid the influence of the random seed on the filter behavior and thus on the conclusions drawn from the results of the experiments. Therefore, it was chosen to do 100 runs per dataset for each setting. The RMSE was calculated for each run and then averaged over 100 runs. The average was determined and also the standard deviation was evaluated to check that the deviation of the filter results of a single realization compared to the mean of the realizations is not unacceptable. This was essential to evaluate for practical reasons. In practice the filter is normally used with one realization (i.e. one ensemble created with a given random seed). The estimate of the filter from this one realization is assumed to be the estimate from the mean of the realizations. It was thus essential that the results of one realization should be as close as possible to the mean.

\subsection{Hindcasting}

The hindcast comparison will start to compare the RMSE of the estimated discharge and the true discharge. Figure 3 shows the results for the different methods.

The EnPF is remarkably better than the EnKF and the $\mathrm{PF}$ (i.e. the mean of the RMSE over 100 runs is small). The EnKF and the EnPF are doing a lot better compared than the PF with a low amount of particles (i.e. 30 members). For a high amount of particles (i.e. 50 members), the PF and the EnPF are doing better than the EnKF which is as expected. In all cases, the EnPF is doing better than both the EnKF and the PF.

Not only is the mean of the RMSE over 100 runs is important but also the standard deviation of the mean of the RMSE over 100 runs as explained earlier. For small
Fig. 3 The top panel shows the mean RMSE of the discharge over 100 runs, and the bottom panel the corresponding standard deviation
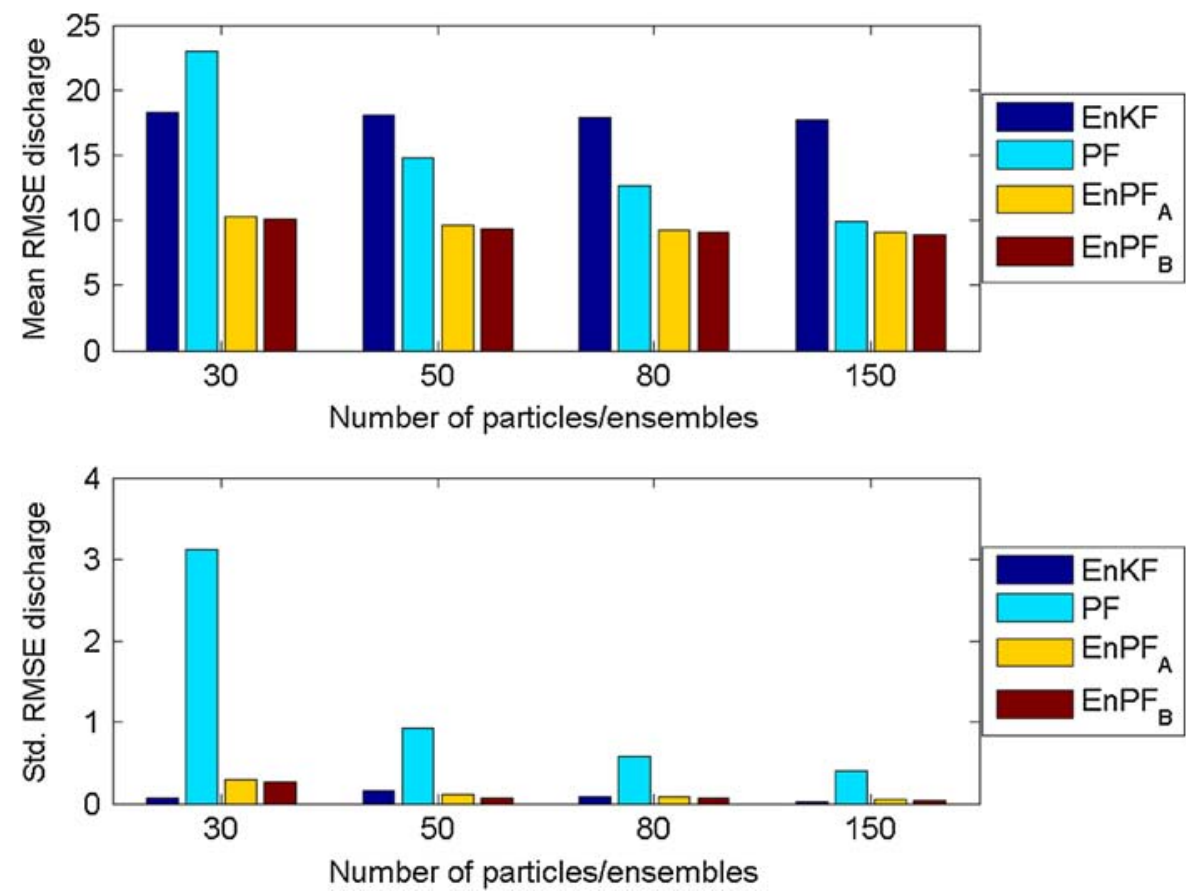
amount of members (i.e. 30 members), the EnPF is significantly smaller than the PF but slightly bigger than the EnKF. For larger amount of members (i.e. 50 and above), the standard deviation of the mean of the RMSE of the EnPF is smaller than both the EnPF and the PF, which proves that the EnPF combinations here proposed is remarkably better than using the EnKF since it adds the advantages of the particle filter in representing other probability density functions rather than the Gaussian and also avoids the disadvantage of the PF in being dependent on the randomness of the realization used.

As a summary and for the hindcast mode, it is thus concluded that the EnPF combinations are showing a significantly improved performance compared to the EnKF and PF. Moreover, the EnPF is less influenced by the random seed of the realization which as explained is an advantage in practice where the filter results are commonly presented from one realization.

\subsection{Forecasting}

The results of this comparison are based on ten runs with different random seeds for the filter. Each run started with 100 days hindcasting to initialize the filter. For a period of 3,000 days forecasts were determined. Forecasts were computed for a maximum of 12 days ahead.

In general all data assimilation methods showed improvements compared to the deterministic model. Figure 4 shows results of forecasts based on the deter- ministic model and one day forecasts when applying the Ensemble Kalman Filter.

Research of this paper focused on combinations between the EnKF and PF. Figure 5 shows results of the forecasting performance of the different filters. Again the mean of the RMSE over 100 runs was calculated.

One can see that the EnKF and the EnPF performance is considerably better compared to the forecast of the PF. Slightly better forecast is observed for the EnPF compared to EnKF. This can be explained due to several reasons among which the first to be mentioned is that no more improvements can be achieved through assimilation than that achieved by the EnKF. As shown in Fig. 4, the EnKF estimate of the EnKF is very close to the true (i.e. green and red lines) while the model is far from the true value. The bad model performance can be due to the coarse time step of the model. Moreover, since the filters are adjusting only the variations in the precipitation due to the discharge measurements, the behavior of the filter is governed by the amount of information present in the discharge measurements that reflects the variations in the precipitations. Including other variables such as uncertain parameters would improve the results.

Results of Fig. 5 take all days of the 3,000 day period into account. One can also focus on the most interesting days, the days with high discharge. Figure 6 displays the RMSE for a 1 day ahead forecast when only days with high discharge are taken into account. The same behavior is encountered.
Fig. 4 Deterministic forecasts and forecasts with the EnKF ( 30 ensemble members) compared to the discharge observations

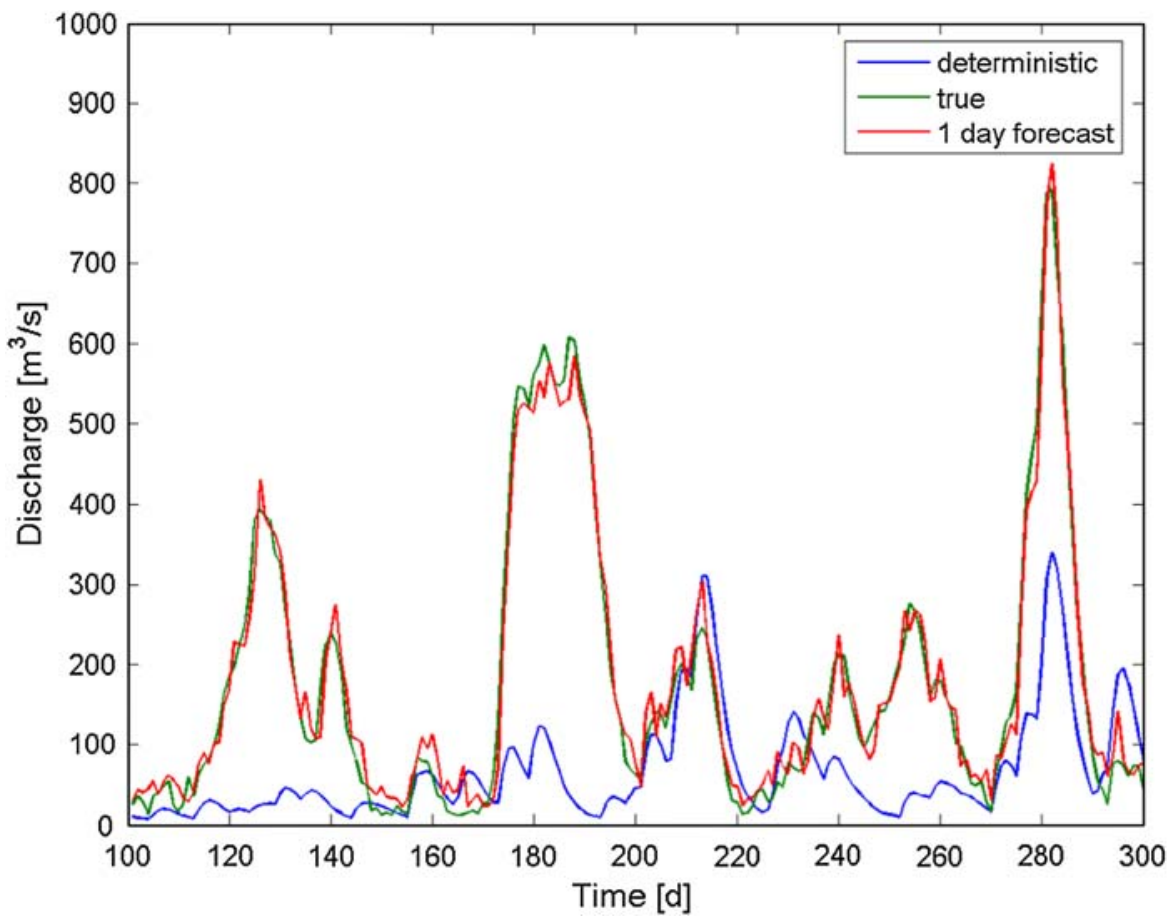




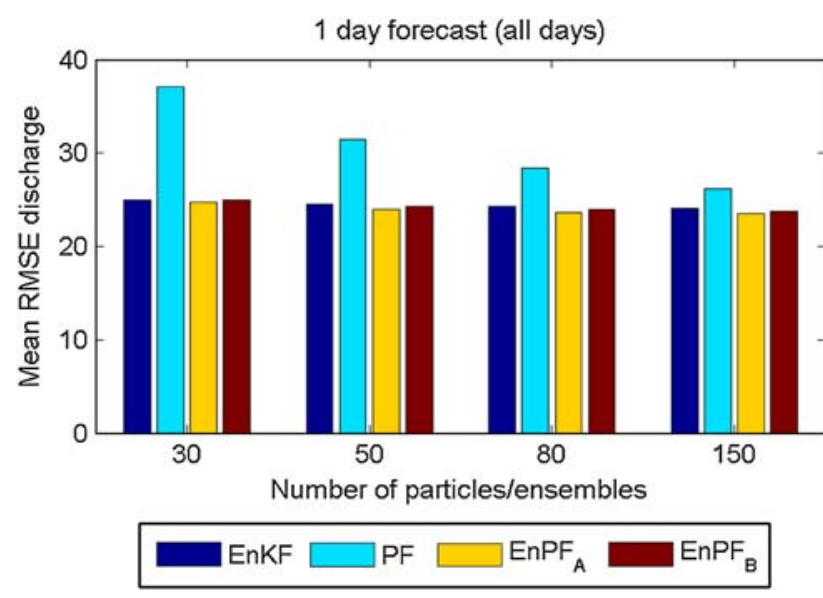

Fig. 5 The RMSE between the 1 day ahead forecast and the observations on the discharge; all values are averages over 100 runs

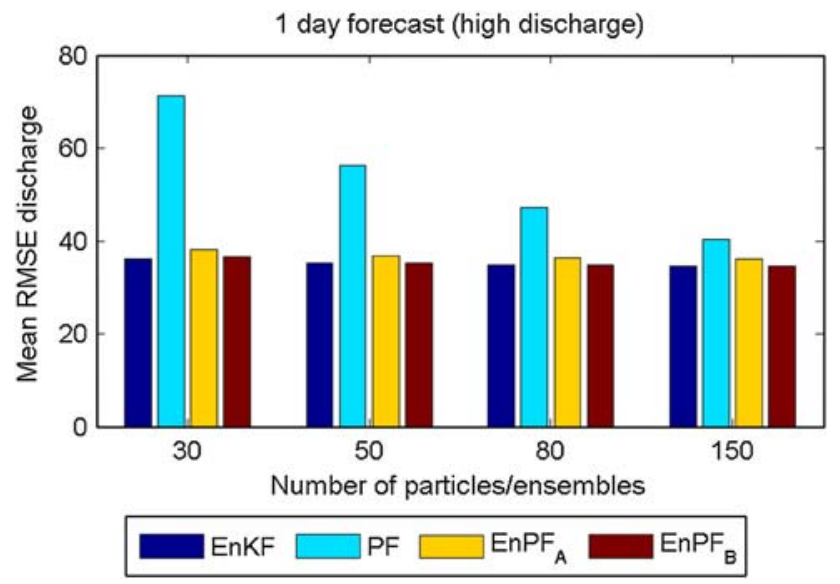

Fig. 6 Same as Fig. 5 only taking days with high discharge into account

\section{Conclusions and recommendations}

The main goal of this paper was to make a combination between the ensemble Kalman filter and the particle filter, in order to combine the best of both. Several suggestions for a combination have been studied. In the synthetic test case that was being used, the Leaf River watershed in combination with the HYMOD rainfall-runoff model, the results show considerable improvements in the hindcast mode and slight improvements were also encountered in the forecast mode. In the hindcast mode, the EnPF combinations are showing a significantly improved performance compared to the EnKF and PF. In the forecast mode, the EnPF performed equally well, or slightly better compared to the EnKF. The EnKF normally uses a simple mean to calculate the output values. For the Particle Filter the weights can be used to calculate a weighted average. The improvement in both combinations of EnPF presented here in the paper lies in the weighted mean used.
Simple modifications to the HYMOD model, such as changing the time step from 1 day to $1 \mathrm{~h}$, would significantly improve the results of the deterministic model and thus the forecast capability of the filters in general. Moreover, since the filters are adjusting only the variations in the precipitation due to the discharge measurements, the behavior of the filter is governed by the amount of information present in the discharge measurements that reflects the variations in the precipitations. Including other variables such as uncertain parameters would improve the results.

In general, combining filters to avoid their disadvantages represents a new challenge in their applications, thus further study to other possible combinations is recommended. Moreover, applications of the proposed EnPF on field data instead of synthetic data would be a logical follow up. It is also recommended to look into possible improvements of the HYMOD model and to study the performance of the proposed combinations when applied to another rainfall-runoff models or completely different practical applications.

\section{References}

Bos and De Vreng (2006) Parameter optimization of the HYMOD model using SCEM-UA and MOSCEM-UA, University of Amsterdam, February

Douc R, Olivier Cappé and Eric Moulines (2005) Comparison of Resampling Schemes for Particle Filtering. Image and Signal Processing and Analysis. ISPA 2005. In: Proceedings of the 4th International Symposium 64-69

Evensen G (1994) Sequential data assimilation with a non-linear quasi-geostrophic model using Monte Carlo methods to forecast error statistics. J Geophys Res 99(C5):10143-10162

Evensen G (2003) The Ensemble Kalman Filter: theoretical formulation and practical implementation. Ocean Dyn 53:343-367

French MN, Bras RL, Krajewski WF (1992) A Monte Carlo study of rainfall forecasting with a stochastic model. J Stoch Hydrol Hydraul 6:27-45

Houtekamer PL, Mitchell HL (2001) A Sequential ensemble Kalman filter for atmospheric data assimilation. Mon Weather Rev 129:123-137

Isard M, Blake A (1998) Conditional density propagation for visual tracking. International Journal of Computer Vision, Volume 29, Number 1/August

Kalman RE (1960) A new approach to linear filtering and prediction problems. Transaction of the ASME, Journal of Basic Engineering, pp. 35-45, March

Koster T, El Serafy G, van den Boogaard HFP, Heemink AW, Mynett A (2004) Input correction in rainfall runoff models using the ensemble Kalman filter, Paper presented at the 4th International Symposium on Environmental Hydraulics, University of Hong Kong, 15-18 December

Mandel J, Beezley JD (2006) Predictor-corrector ensemble filters for the data assimilation of sparse data into high dimensional nonlinear systems, University of Colorado at Denver and Health Sciences Center, June

Moore RJ (1985) The probability-distributed principle and runoff production at point and basin scale. J Hydrol Sci 30(2):273-297 
Shu Q, Kemblowski MW, McKee M (2005) An application of ensemble Kalman filter in integral balance subsurface modeling. Stoch Environ Res Risk Anal 19:361-374. doi:10.1007/s00477005-0242-8
Weerts AH, El Serafy GYH (2006) Particle filtering and ensemble Kalman filtering for state updating with hydrological conceptual rainfall-runoff models. Water Resour Res 42(9):W09403. doi: 10.1029/2005WR004093 